\title{
Assessment of fracture risk assessment tools in care home residents
}

\author{
Author: Felix Ihama ${ }^{\mathrm{A}}$
}

\section{Introduction}

Fragility fractures are common in care home residents. National guidelines recommend risk assessment to allow initiation of prophylactic measures. Currently available risk assessment tools have been tested in community-dwelling adults, but not in care home residents. It is possible that one or more of the existing tools are also practicable in this population.

\section{Aim}

The aim of this project was to identify fracture risk assessment tools that are usable in care home residents and to determine which is the most suitable for use in this population.

\section{Objectives}

$>$ Conduct a systematic literature review of the existing fragility risk assessment tools and select those that can be used in care home residents.

> Then undertake an observational pilot study of the fragility risk assessment in a cohort of care home residents.

\section{Methods}

> A literature search was performed by a combination of electronic and manual literature searches, and studies of assessment tools potentially usable in a care home population were selected and assessed based on content and quality criteria. The search was updated on 12 August 2019.

$>$ A cohort observational study was conducted in 18 care homes in Boston, Lincolnshire, England.

\section{Results}

> In the systematic review, 33 fragility tools were identified and four were potentially practicable in care home residents. These were FRAX, QFractureScores, Garvan nomogram and body mass index (BMI). The updated search identified a fifth measure, microribonucleic acid (miRNA). However, this was not implemented.

\footnotetext{
Author: ${ }^{A}$ United Lincolnshire Hospitals NHS Trust, Lincoln, UK
}

> In the feasibility study, 217 (35\%) participants out of 618 residents in the 18 care homes were enrolled. Of the 217 participants, 147 (68\%) had mental capacity and $70(32 \%)$ did not because of the difficulty in obtaining informed consent from the consultees in residents without mental capacity.

\section{Discussion}

The systematic literature review identified many fragility risk assessment tools, but only four were potentially practicable in a care home population. Recruitment to the observational study was restricted mainly to residents who possessed mental capacity, because it was difficult to obtain consultee consent in this setting. The statistically significant odds ratios for the outcomes were: BMI falls 0.952, standard error (SE) 0.021 ( $p=0.015)$, fractures 0.868 , SE $0.073(p=0.024)$, combined falls and fractures 0.868 , SE $0.073(p=0.024)$. Low BMI and history of dementia were identified as the risk factors for falls, fractures and combined falls and fractures in the cohort. A BMI of $25 \mathrm{~kg} / \mathrm{m}^{2}$ or less had the highest sensitivity of $74.5 \%$ for falls. Of the 10 incident fractures, $40 \%$ occurred in the participants who had dementia.

\section{Conclusions}

BMI was the best predictor of falls, fractures and combined falls and fractures, but the associations were weak. Of the 10 incident fractures, $40 \%$ were observed in participants who had dementia despite the small representation of this group, thus dementia is a strong risk factor for fractures in this cohort. A fully powered and representative study is unlikely to be feasible if individual consent is required, as the majority of care home residents do not have mental capacity, and legal representative consent is difficult to obtain in this setting.

\section{Conflicts of interest}

None declared. 